TITLE:

\title{
Neutron spin-echo studies on dynamic and static fluctuations in two types of poly(vinyl alcohol) gels
}

\section{$\operatorname{AUTHOR}(\mathrm{S}):$}

Kanaya, T; Takahashi, N; Nishida, K; Seto, H; Nagao, M; Takeda, T

\section{CITATION:}

Kanaya, T ...[et al]. Neutron spin-echo studies on dynamic and static fluctuations in two types of poly(vinyl alcohol) gels. Physical Review E 2005, 71(1): 011801.

\section{ISSUE DATE:}

2005-01

URL:

http://hdl.handle.net/2433/39909

RIGHT:

Copyright 2005 American Physical Society 
PHYSICAL REVIEW E 71, 011801 (2005)

\title{
Neutron spin-echo studies on dynamic and static fluctuations in two types of poly(vinyl alcohol) gels
}

\author{
T. Kanaya ${ }^{1, *}$ N. Takahashi, ${ }^{1}$ K. Nishida, ${ }^{1}$ H. Seto, ${ }^{2}$ M. Nagao, ${ }^{3}$ and T. Takeda ${ }^{4}$ \\ ${ }^{1}$ Institute for Chemical Research, Kyoto University, Uji, Kyoto-fu 611-0011, Japan \\ ${ }^{2}$ Department of Physics, Faculty of Science, Kyoto University, Kyoto, 606-5802, Japan \\ ${ }^{3}$ Institute for Solid State Physics, The University of Tokyo, 106-1 Shirakata, Tokai, Ibaraki-ken 319-1106, Japan \\ ${ }^{4}$ Faculty of Integrated Arts and Sciences, Hiroshima University, Higashihiroshima, Hiroshima-ken 192-0397, Japan
}

(Received 16 July 2004; published 7 January 2005)

\begin{abstract}
We report neutron spin-echo measurements on two types of poly(vinyl alcohol) (PVA) gels. The first is PVA gel in a mixture of dimethyl sulfoxide (DMSO) and water with volume ratio 60/40, and the second is PVA gel in an aqueous borax solution. The observed normalized intermediate scattering functions $I(Q, t) / I(Q, 0)$ are very different between them. The former $I(Q, t) / I(Q, 0)$ shows a nondecaying component in addition to a fast decay, but the latter does not have the nondecaying one. This clearly indicates that the fluctuations in the former PVA gel consist of static and dynamic fluctuations whereas the latter PVA gel does include only the dynamic fluctuations. The dynamic fluctuations of the former and latter gels have been analyzed in terms of a restricted motion in the network and Zimm motion, respectively, and the origins of these motions will be discussed.
\end{abstract}

DOI: 10.1103/PhysRevE.71.011801

PACS number(s): 61.12.Ex, 82.70.Gg, 61.41.+e

\section{INTRODUCTION}

A polymer gel is a three-dimensional network swollen by a solvent and shows many interesting properties, and hence many investigations have been performed aiming industrial applications of polymer gels such as shock absorbers, drag delivery systems, biomedical materials, and actuators [1-3]. In order to give a basis to these applications, fundamental studies on structure and dynamics are essential. Recently extensive investigations have been carried out on structure of polymer gels using scattering techniques. Small-angle x-ray and neutron scattering experiments [4] have revealed characteristic length scales related to the network size and/or thermal fluctuations on a spatial scale from $\mathrm{nm}$ to several tens $\mathrm{nm}$. Further studies have determined that liquid-liquid phase separation often occurs in polymer gel systems on the $\mu \mathrm{m}$ scale during and after the gel formation process [5,6]. This has been observed using a light scattering experimentation $[4,7]$. From these various observations polymer gels are now established as inhomogeneous and hierarchic structure on a wide spatial scale from $1 \AA$ to $10 \mu \mathrm{m}$. Recent speckle experiments on polymer gels have directly showed the inhomogeneity of polymer gels $[7,8]$.

Poly(vinyl alcohol) (PVA) is one of the most interesting gel-forming polymers due to its water solubility and biocompatibility. This polymer forms physically cross-linked gels in many solvents and also chemically cross-linked gels through a reaction with many kinds of cross-linkers. The resultant gels are very different in appearance as well as in properties. We have extensively studied structure of PVA physical gels formed in a mixture of dimethy sulfoxide (DMSO) and water with volume ratio $60 / 40$ on a wide spatial scale from

\footnotetext{
*Corresponding author. FAX: +81-(0)774-38-3146. Electronic address: kanaya@scl.kyoto-u.ac.jp
}

$1 \AA$ to $10 \mu \mathrm{m}$ using wide-angle neutron scattering (WANS) [9,10], small-angle neutron scattering (SANS) [9-11], light scattering (LS) $[10,12]$, and ultrasmall-angle neutron scattering (USANS) [13]. These studies revealed that there are many kinds of fluctuations or inhomogeneity on a wide spatial scale. However, whether the fluctuations are static (or frozen) or dynamic has not been determined since scattering methods such as small-angle x-ray scattering (SAXS), SANS, and LS do not detect the individual fluctuations. To understand the response of the PVA gels to external fieldsi.e., temperature, $p \mathrm{H}$, and electric field-it is necessary to investigate the gel dynamics.

Dynamic studies on polymer gels have been mainly involved dynamic light scattering (DLS) experimentation $[14,15]$. Pioneering research by Tanaka et al. [16] revealed the so-called gel mode, and many other groups found the inhomogeneous dynamics of polymer gels [7]. The LS studies give information on the dynamics of gels on a spatial scale of $\mu \mathrm{m}$. As for the PVA gel in a mixture of DMSO and water $(60 / 40)$, we do not know the microscopic dynamics of the gel. In this paper, we have investigated the dynamics of the PVA gel, particularly focusing on a spatial scale of $\mathrm{nm}$ to tens $\mathrm{nm}$. For this purpose we have employed a neutron spinecho (NSE) technique [17] because it is the only method to directly investigate the gel dynamics on this spatial scale [18-21].

In the previous study [20], we have shown that the NSE technique is very powerful to study the dynamics of the PVA gels in a mixture of DMSO and water. In this work, we performed further measurements on the PVA gel to get final conclusions. Furthermore, we have also investigated the dynamics of PVA in aqueous borax solutions for comparison. It is well known that the solution viscosity drastically increases by slight borax addition above a certain critical PVA concentration [22]; however, it is dependent on molecular weight. The complex formation between PVA hydroxy groups and a 
borate anion determines the viscosity, which plays a role of transient cross-linking point [23]. The dynamics of this gel has been extensively studied using DLS [24-27] and mechanical spectroscopy [28-30] while it has never been reported so far on dynamics on the nm scale. According to mechanical measurements the lifetime of the complex (the transient cross-linking point) is about $0.1 \mathrm{~s}$ while the crosslinking point of the PVA gel in a mixture of DMSO and water $(60 / 40)$ is a crystallite with infinite lifetime unless melting upon heating. We therefore expect surely distinct differences in dynamics to arise between the two PVA gels.

\section{EXPERIMENT}

\section{A. Materials}

In this work, we studied PVA gels formed in a mixture of dueterated dimethyl sulfoxide (DMSO- $\mathrm{d}_{6}$ ) and heavy water $\left(\mathrm{D}_{2} \mathrm{O}\right)$ with volume ratio of $60 / 40$ and PVA gels in an aqueous borax solution. The former was prepared by dissolving atactic PVA with weight-average degree of polymerization $D P=1730$ into the mixture at $130^{\circ} \mathrm{C}$ to have a homogeneous solution. This PVA has molecular weight distribution $M_{\mathrm{w}} / M_{\mathrm{n}}=1.98$, where $M_{\mathrm{w}}$ and $M_{\mathrm{n}}$ are the weight- and number-average molecular weights, respectively, and the degree of saponification is $99.8 \%$. The homogeneous solution was quenched to $25^{\circ} \mathrm{C}$ for gelation. The PVA concentration $C_{\mathrm{p}}$ was $5.0 \mathrm{~g} / \mathrm{dl}$ for the measurements. For the latter gel, we used atactic PVA with $D P=600, M_{\mathrm{w}} / M_{\mathrm{n}}=2.0$, and degree of saponification of $87.9 \%$. This PVA was dissolved in a heavy water $\left(\mathrm{D}_{2} \mathrm{O}\right)$ at $90{ }^{\circ} \mathrm{C}$, and solution of sodium borate $\left(\mathrm{Na}_{2} \mathrm{~B}_{4} \mathrm{O}_{7} 10 \mathrm{H}_{2} \mathrm{O}\right)$ was also dissolved in heavy water separately. The two solutions were mixed to obtain the desired concentration $C_{\mathrm{p}}$. The concentration of sodium borate was always adjusted to be half of PVA by weight, corresponding to the molar ratio of sodium borate of 0.058 . The solutions were kept at $25^{\circ} \mathrm{C}$ for $24 \mathrm{~h}$ for gelation.

\section{B. Measurements}

Small-angle neutron scattering measurements were performed with SANS-U diffractometer [31] at the JRR-3M reactor in Tokai. The SANS-U is installed at the end of a cold neutron guide $(\mathrm{C}-1)$. The neutron wavelength $\lambda$ in this measurement was $7 \AA$ with a dispersion $\Delta \lambda / \lambda$ of $10 \%$. The diameter of the irradiated neutron beam was $5 \mathrm{~mm}$ and the scattered neutrons are detected by a two-dimensional position-sensitive detector having $65 \times 65 \mathrm{~cm}^{2} \quad(128$ $\times 128$ pixels) area. In the measurements, we covered a range of magnitude of scattering vector $Q(=4 \pi \sin \theta / \lambda, \lambda$ and $2 \theta$ being the neutron wavelength and scattering angle, respectively) from 0.008 to $0.15 \AA^{-1}$.

In the SANS measurements, incoherent scattering contributions from hydrogen atoms of the gel samples were evaluated by measuring the scattering intensity from hydrogenated methanol dissolved in the deuterated solvent so that the hydrogen concentration would be the same as that of a given gel sample.

Neutron spin-echo measurements were carried out using NSE spectrometer [32] installed at a cold neutron beam port
C2-2 in JRR-3M reactor, Tokai. In the NSE measurements the normalized intermediate scattering function $I(Q, t) / I(Q, 0)$ is obtained. In the present measurements we used neutrons with wavelength $\lambda=7.1 \AA$ and dispersion of $\Delta \lambda / \lambda=18 \%$, covering the Fourier time from 0.08 to $15 \mathrm{~ns}$. The $Q$ range covered in the experiment was $0.02-0.13 \AA^{-1}$. The resolution function was estimated using scattering from graphite foils at each scattering angle. The experiments were carried out at $25^{\circ} \mathrm{C}$ for all the gels.

\section{RESULTS AND DISCUSSION}

\section{A. PVA gel formed in a mixture of DMSO and water with volume ratio $60 / 40$}

First, we focus on static structure of the PVA gel formed in a mixture of DMSO and water with volume ratio 60/40. In previous papers [9-13], we have studied the structure of the PVA gel and its formation process at $25^{\circ} \mathrm{C}$ in a wide spatial scale from $1 \AA$ to $10 \mu \mathrm{m}$ using wide-angle neutron scattering, small-angle neutron scattering, ultrasmall-angle neutron scattering, and light scattering techniques. LS [12] and USANS [13] studies revealed that spinodal decomposition (SD) type liquid-liquid phase separation occurs before the network formation and the solution is separated into polymer-rich and polymer-poor phases. SANS $[9,11]$ and WANS [9] studies showed that polymer network is formed in the polymer-rich phase and the cross-linking points of the network are crystallites, and the distance between the nearest-neighboring crystallites is approximately $200 \AA$ and the size is approximately $70 \AA$ in radius. On the basis of these observations, the Porod's law $\left[I(Q) \sim Q^{-4}\right]$ observed in the $Q$ range between 0.05 and $0.1 \AA^{-1}$ has been assigned to smooth surface of the crystallites. This $Q$ range almost corresponds to the $Q$ range in the present NSE measurements, implying that we mainly observe the dynamics of the crosslinking points or the crystallites in the gel.

In this work, we again performed SANS measurements on the same PVA gel as the NSE measurements and the observed SANS intensity is shown in Fig. 1 as a function of $Q$ to confirm the Porod's law. Intensity measurements were also done using the NSE spectrometer and the result is plotted in Fig. 1. The agreement of the $Q$ dependence of the intensity between the SANS and NSE measurements is good, depicting that the intensity measurements by NSE were satisfactory.

In the SANS measurements we observe the particle density fluctuations or the concentration fluctuations in the PVA gel. It is expressed in the atomic coordinate expression, which can be easily reduced to the continuum expression [33]

$$
I(\boldsymbol{Q}, t=0)=N^{-1} \sum_{i=1}^{N} \sum_{j=1}^{N}\left\langle\exp \left[-i \boldsymbol{r}_{i}(0) \boldsymbol{Q}\right] \exp \left[i \boldsymbol{r}_{j}(0) \boldsymbol{Q}\right]\right\rangle,
$$

where $N$ is number of scattering elements, $\boldsymbol{r}_{\mathrm{i}}(0)$ and $\boldsymbol{r}_{\mathrm{j}}(0)$ are the positions of the $i$ th and $j$ th elements, respectively, and $\langle\cdots\rangle$ means the ensemble average. This corresponds to a time correlation function of $\exp [-i r(t) Q]$ at $t=0$. In the NSE measurements, on the other hand, we can observe the time evo- 


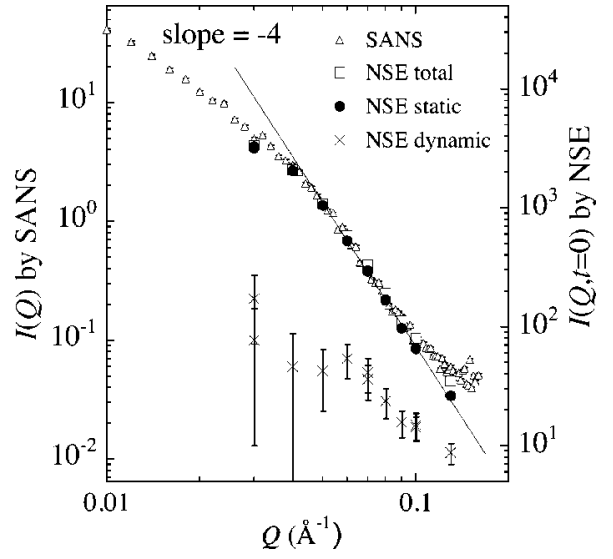

FIG. 1. Small-angle neutron scattering intensity of PVA gel in a mixture of DMSO and water $(60 / 40)$. $(\triangle)$ measured by SANS spectrometer, $(\square)$ measured by NSE spectrometer, $(\bullet)$ static (frozen) component in NSE total scattering intensity, and $(\times)$ dynamic component in NSE total scattering intensity.

lution of fluctuations or the so-called coherent intermediate scattering function $I(Q, t)[33]$

$$
I(\boldsymbol{Q}, t)=N^{-1} \sum_{i=1}^{N} \sum_{j=1}^{N}\left\langle\exp \left[-i \boldsymbol{r}_{i}(0) \boldsymbol{Q}\right] \exp \left[i \boldsymbol{r}_{j}(t) \boldsymbol{Q}\right]\right\rangle .
$$

If the fluctuations are time dependent, $I(Q, t)$ decays with time. On the other hand, if they are frozen, it is independent of time remaining constant. The former and the latter are called static (or frozen) and dynamic fluctuations, respectively.

In order to see the time dependent and/or time independent fluctuations we have evaluated the normalized intermediate scattering function $I(Q, t) / I(Q, 0)$ using the NSE spectrometer in the $Q$ range from 0.03 to $0.12 \AA^{-1}$. The observed $I(Q, t) / I(Q, 0)$ is shown in Fig. 2 at $Q=0.04,0.07,0.10$, and $0.12 \AA^{-1}$. In a short time region below about $3 \mathrm{~ns}$, $I(Q, t) / I(Q, 0)$ decays rapidly, while it does not decay and remains constant in the longer time range. The observed intermediate scattering function can be phenomenologically described by

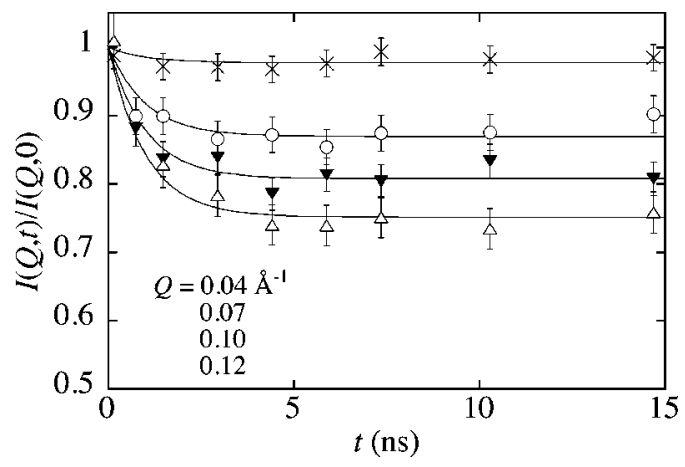

FIG. 2. Normalized intermediate scattering function $I(Q, t) / I(Q, 0)$ of PVA gel in a mixture of DMSO and water (60/40). (×) $Q=0.04 \AA^{-1}$, (○) $0.07 \AA^{-1}$, ( $\left.\mathbf{\nabla}\right) 0.10 \AA^{-1},(\triangle)$ $0.12 \AA^{-1}$. Solid curves are the results of fit with Eq. (3) (see text).

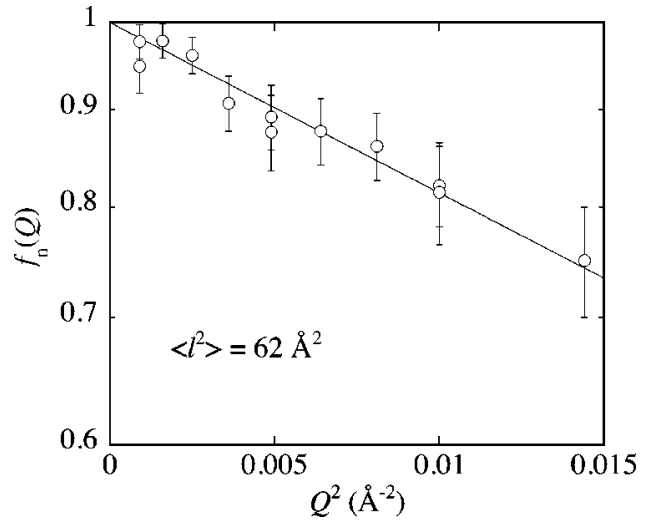

FIG. 3. Logarithm of nondecaying component fraction $f_{\mathrm{n}}(Q)$ in $I(Q, t) / I(Q, 0)$ of PVA gel in a mixture of DMSO and water $(60 / 40)$ as a function of $Q^{2}$.

$$
I(Q, t) / I(Q, 0)=f_{n}(Q)+\left[1-f_{n}(Q)\right] F(Q, t),
$$

where $f_{\mathrm{n}}(Q)$ is a fraction of the nondecaying component and $F(Q, t)$ is a generalized decay function. Assuming that the decay function could be expressed by a single-exponential function, we fitted Eq. (3) to the observed intermediate functions to evaluate the relaxation time $\tau_{\mathrm{f}}$ and the fraction of the nondecaying component $f_{\mathrm{n}}(Q)$ as a function of $Q$. The results are shown by solid curves in Fig. 2, showing good agreement with the observed ones within the experimental accuracy. The fractions of the nondecaying component $f_{\mathrm{n}}(Q)$ and the rest, $1-f_{\mathrm{n}}(Q)$, correspond to those of the static and dynamic fluctuations, respectively. The observed total SANS intensity $I(Q)$ is then divided into the static $f_{\mathrm{n}}(Q) I(Q)$ and dynamic fluctuations $\left[1-f_{\mathrm{n}}(Q)\right] I(Q)$, and the results are shown in Fig. 1. This clearly suggests that the total SANS intensity is governed mainly by the static fluctuations in the PVA gel, indicating that the molecular motions in the PVA gel are almost frozen, at least in the present $Q$ range.

The only motion observed in the present $Q$ range is the fast decay in the time region below about $3 \mathrm{~ns}$. We now consider what motion is observed in the NSE measurement. The fact that there exists a nondecaying component (or the elastic component) means that the motion is a localized one in a confined space. In the gel, the motion of cross-linking points is possibly restricted in the network. If we assume that for asymptotic times the probability of finding a crosslinking point at a distance from its equilibrium position is described by a Gaussian function, the fraction of nondecaying component (or the elastic component) is given by

$$
f_{n}(Q)=\exp \left(-\left\langle l^{2}\right\rangle Q^{2} / 3\right) .
$$

Here $\left\langle l^{2}\right\rangle$ is the mean-square displacement of the scattering object. According to Eq. (4), we plotted the logarithm of the nondecaying component fraction $f_{\mathrm{n}}(Q)$, which is of the fast decay counter part, as a function of $Q^{2}$ in Fig. 3, giving a well-fitted straight line. This may suggest that we mainly observed the motion of cross-linking points confined in the network. From the slope of the straight line in Fig. 3, we have estimated the root-mean-square displacement $\sqrt{\left\langle l^{2}\right\rangle}$ to be $7.9 \AA$. This is an analysis based on the assumption that 


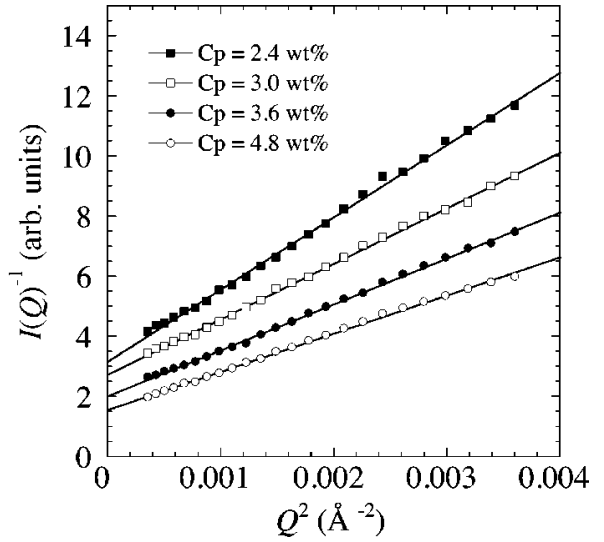

FIG. 4. Inverse of small-angle neutron scattering intensity of

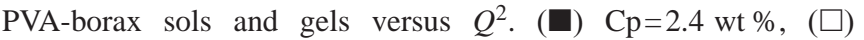
$3.0 \mathrm{wt} \%$, (○) $3.6 \mathrm{wt} \%$, and $(\bigcirc) 4.8 \mathrm{wt} \%$.

the motion observed in the experiment is a motion confined in a finite space. However, there are other possibilities. One of them is the motion of amorphous tie chains between the cross-linking points, which must be described the Zimm mode. The other is rotational diffusive motion of the crosslinking point. At the moment, however, we have no final conclusions because of lack of knowledge on the characteristics of the fast decay, especially on the $Q$ dependence of the decay time.

\section{B. PVA gels in an aqueous borax solution}

In the second experiment we have studied structure and dynamics of PVA in an aqueous borax solution. The viscosity of the aqueous solution with the PVA concentration $C_{\mathrm{p}}$ $=2.4 \mathrm{wt} \%$ is about $2 \times 10^{-3} \mathrm{~Pa} \mathrm{~s}$, this abruptly begins to increase with the polymer concentration at around $C_{\mathrm{p}}$ $=3.0 \mathrm{wt} \%$, and the viscosity is about $4 \times 10^{2} \mathrm{~Pa} \mathrm{~s}$ at $C_{\mathrm{p}}$ $=4.8 \mathrm{wt} \%$, being more than 5 orders of magnitude larger than that of $C_{\mathrm{p}}=2.4 \%$. Systematic measurements of viscosity of the PVA gels in aqueous borax solutions have been performed by Takada et al. $[26,27]$. In this paper, we will refer to the aqueous borax solutions of PVA with the concentration below and above $3.0 \mathrm{wt} \%$ as PVA-borax sol and gel, respectively.

In the aqueous borax solutions of PVA the gelation process occurs owing to cross-linking of PVA chains through hydrogen bonds between hydroxyl groups on PVA chains and a borate anion, and the cross-linking points are exchangeable and the gel can flow extremely slowly, and hence the PVA-borax gel is in fact not a gel. In this paper, though, we refer to it as a gel for distinction between the solution. The mechanical measurements on this gel [28-30] predicted that the relaxation time of the cross-linking point is approximately $0.1 \mathrm{~s}$. This gives basis for interpretation of the results on dynamics by NSE measurements.

SANS measurements were performed on the PVA-borax sols and gels at the PVA concentration $C_{\mathrm{p}}$ of 2.4, 3.0 3.6, and $4.8 \mathrm{wt} \%$, and the inverse of the observed intensity $I(Q)$ is plotted as a function of $Q^{2}$ in a $Q$ range of $0.016-0.06 \AA^{-1}$ in Fig. 4: the so-called Ornstein-Zernike plot. As seen in the figure, the straight lines are obtained in this plot, showing that the scattering intensity displays clear agreement with the Ornstein-Zernike expression,

$$
I(Q)=\frac{I(0)}{1+\xi^{2} Q^{2}},
$$

where $\xi$ and $I(0)$ are a correlation length and the scattering intensity at $Q=0$, respectively. The correlation lengths evaluated from the straight lines in Fig. 4 are 27.5, 26.0, 27.4, and $28.4 \AA$ for $C_{\mathrm{p}}=2.4,3.0,3.6$, and $4.8 \mathrm{wt} \%$, respectively. The correlation length is almost independent of the PVA concentration, at least in the present concentration range. In the beginning of the experiment we expected to observe the network size in the gel, which must decrease with increasing the concentration according to $\xi \sim C_{p}^{-1 / 3}$. In this measurement the concentration was from 2.4 to $4.8 \mathrm{wt} \%$, meaning that correlation length decreases by a factor of $0.80\left(=2^{-1 / 3}\right)$. The correlation length is $27.5 \AA$ at $C_{\mathrm{p}}=2.4 \%$, and if we observe the network size, the correlation length should be $22 \AA$ at $C_{p}$ $=4.8 \%$; however, the observed value was $28.4 \AA$. This discrepancy is certainly outside the error of the measurement. At the moment we have no conclusive evidence, but one possibility is that the correlation length is related to the size of the cross-linking points. In fact, the size of the crosslinking point is about $20 \AA$ from the molecular model.

Normalized intermediate scattering functions $I(Q, t) / I(Q, 0)$ measured by the NSE spectrometer are shown in Figs. 5 for the PVA-borax sol with $C_{\mathrm{p}}=2.4 \mathrm{wt} \%$ and the PVA-borax gel with $C_{\mathrm{p}}=4.8 \mathrm{wt} \%$, respectively. In contrast to $I(Q, t) / I(Q, 0)$ of the PVA gel formed in a mixture of DMSO and water (see Fig. 2), they appear to decay to zero at infinite time, even in the gel.

The PVA-borax sol is a solution and it is known that the Zimm model [34] can well describe polymer motions in solutions [35]. In order to analyze these intermediate scattering functions we employed the Zimm model, which is a beadspring model with hydrodynamic interactions. The coherent intermediate scattering function was calculated by de Gennes and Dubois-Violette [36], which is given by

$$
\begin{gathered}
\frac{I(\mathbf{Q}, t)}{I(\mathbf{Q}, 0)}=\int_{0}^{\infty} d u \exp \left\{-u-\left(\Gamma_{Z} t\right)^{2 / 3} h\left[\frac{u}{\left(\Gamma_{Z} t\right)^{2 / 3}}\right]\right\}, \\
h(y)=\frac{4}{\pi} \int_{0}^{\infty} d x \frac{\cos x^{2}}{x^{3}}\left[1-\exp \left(-y^{-3 / 2} x^{3}\right)\right], \\
\Gamma_{Z}=\frac{1}{6 \pi} \frac{k_{B} T}{\eta_{s}} Q^{3},
\end{gathered}
$$

where $\Gamma_{z}$ is the Zimm decay rate and $\eta_{s}$ is the solvent viscosity. From Eq. (6) it is evident that the coherent intermediate scattering function is scaled by $\left(\Gamma_{z} t\right)^{2 / 3}$ or $\left(Q^{3} t\right)^{2 / 3}$. The observed intermediate scattering functions are plotted against the so-called Zimm time $\left(Q^{3} t\right)^{2 / 3}$ for the PVA-borax sol and gel in Figs. 6, respectively. The data points fall on the master curve theoretically calculated from Eq. (6), suggesting that the Zimm scaling works well for the sol and even for the gel. Note that to determine if the Zimm model is suitable or not 

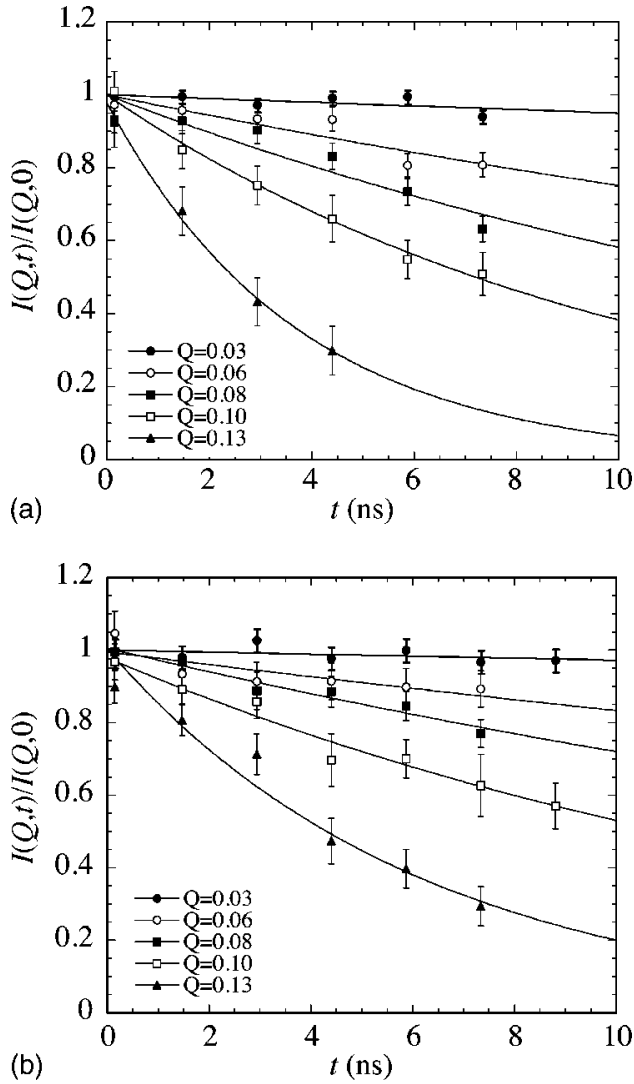

FIG. 5. Normalized intermediate scattering functions $I(Q, t) / I(Q, 0)$ of (a) PVA-borax sol with $C_{\mathrm{p}}=2.4 \mathrm{wt} \%$ and (b) gel with $C_{\mathrm{p}}=4.8$ wt \%. (○) $Q=0.03 \AA^{-1},(\bigcirc) 0.06 \AA^{-1}$, (田 $0.08 \AA^{-1}$, (口) $0.10 \AA^{-1}$, and $(\mathbf{\Delta}) 0.13 \AA^{-1}$. Solid lines are drawn as a guide by eye.

in the low- $Q$ range remains difficult since the range of Zimm time is too narrow. The ratio of the decay rate $\Gamma_{z}$ of the gel to the sol is only 2.2 although the viscosity of the gel is more than 5 orders of magnitude larger than that of the sol. Surprisingly the PVA-borax gel appears as the sol, with respect to the intermediate scattering function, at least in the present $Q$ range of $0.02-0.12 \AA^{-1}$. Why can the Zimm model describe the dynamics of the gel whose viscosity is more than 5 orders of magnitude larger than that of the sol.

In order to consider this problem, we have examined the $Q$ dependence of the first cumulant or the initial decay rate of $\ln [I(Q, t) / I(Q, 0)] \quad(=-d\{\ln [I(Q, t) / I(Q, 0)]\} / d t)$. For the evaluation, we used the data points in the whole time range in the low- $Q$ range below $0.06 \AA^{-1}$, while we used the data points up to $6,4.5$, and 3 ns for $Q=0.08 \AA, 0.10 \AA^{-1}$, and above, respectively. Note that the initial decay rate at $Q$ $=0.03 \AA^{-1}$ is not reliable because the $I(Q, t) / I(Q, 0)$ hardly decays in the present time region. In Fig. 7, the initial decay rate $\Gamma_{\mathrm{i}}$ is plotted versus $Q$ for the PVA-borax sol and gel in a double logarithmic form. In the $Q$ range above about a certain critical value $Q_{\mathrm{c}}$ indicated by arrows in the figure, the $\Gamma_{\mathrm{i}}$ is proportional to $Q^{3}$ for both the sol and gel. This agrees with the $Q$ dependence of the Zimm decay rate $\Gamma_{\mathrm{z}}$ [see Eq. (6)], confirming again that the Zimm model is appropriate to describe the dynamics of both the sol and gel, at least above $Q_{\text {c }}$. On the other hand, the initial decay $\Gamma_{\mathrm{i}}$ is proportional to
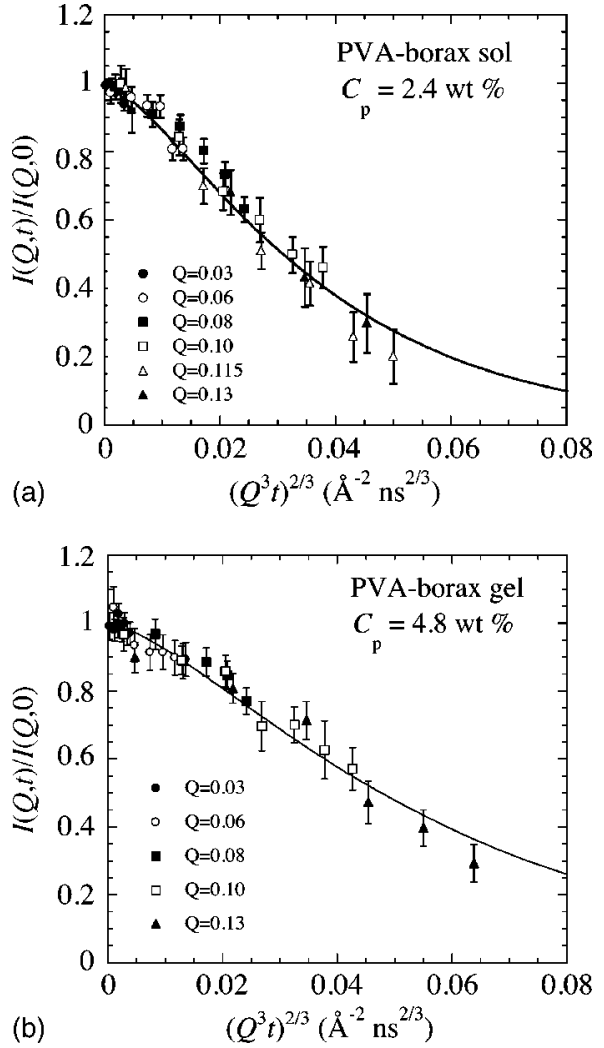

FIG. 6. Zimm scaling of normalized intermediate scattering functions $I(Q, t) / I(Q, 0)$ of (a) PVA-borax sol with $C_{p}=2.4 \mathrm{wt} \%$ and (b) gel with $C_{p}=4.8$ wt \% . (○) $Q=0.03 \AA^{-1},(\bigcirc) 0.06 \AA^{-1},(\boldsymbol{\square})$ $0.08 \AA^{-1},(\square) 0.10 \AA^{-1}$, and $(\mathbf{A}) 0.13 \AA^{-1}$. Solid curves are the Zimm master curve calculated from Eq. (6) (see text).

$Q^{2}$ in the $Q$ range below the critical value $Q_{\text {c }}$. This must be the so-called gel mode [16], suggesting that the Zimm mode is not appropriate in the low- $Q$ range below $Q_{\text {c }}$. The gel mode is a propagating mode on elastic gel network, permitting over a range larger than the mesh size of the network, which can be approximately evaluated from the critical $Q_{\mathrm{c}}$ at

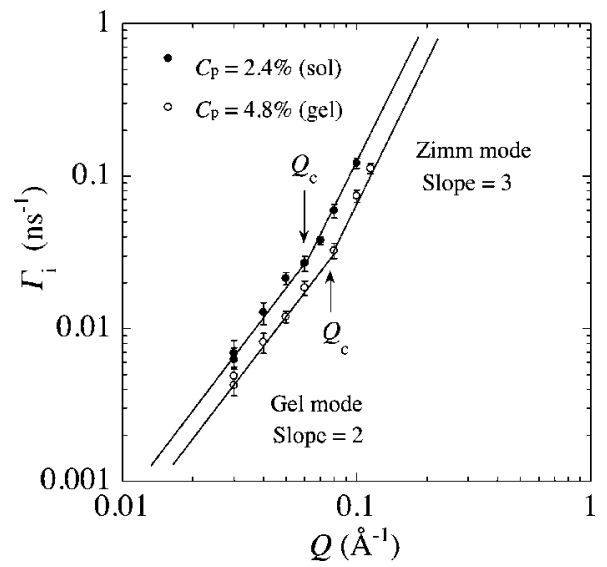

FIG. 7. $Q$ dependence of first cumulant $\Gamma_{\mathrm{i}}$ (initial decay of $\ln [I(Q, t) / I(Q, 0)])$ for PVA-borax sol with $C_{\mathrm{p}}=2.4 \mathrm{wt} \%(\bigcirc)$ and gel with $C_{\mathrm{p}}=4.8 \mathrm{wt} \%(\bigcirc)$. Error bars in the figure are evaluated from the counting statistics, but do not include the error in the decay fit. 


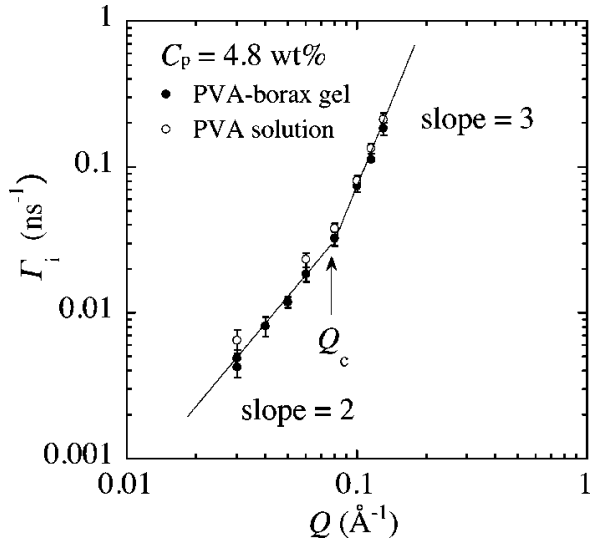

FIG. 8. $Q$ dependence of first cumulant $\Gamma_{\mathrm{i}}$ (initial decay of $\ln [I(Q, t) / I(Q, 0)])$ for PVA-borax gel with $C_{\mathrm{p}}=4.8 \mathrm{wt} \%(\bullet)$ and PVA solution with $C_{\mathrm{p}}=4.8 \mathrm{wt} \%(\bigcirc)$. Note that the solution does not include borax.

the crossover between the Zimm and gel modes. The critical values $Q_{\mathrm{c}}$ are 0.060 and $0.075 \AA^{-1}$ for the borax sol and gel, respectively, result in the mesh size $\left(2 \pi / Q_{c}\right)$ of 105 and $83.8 \AA$, respectively. The ratio of $Q_{\text {c }}$ of the sol to gel is 1.25 , which is very close to $\sqrt[3]{2}$. This means that the mesh size of the gel network is proportional to the $-1 / 3$ power of the PVA concentration, suggesting that the network structure of the sol and gel is rather homogeneous. The fact that the gel mode is observed in both the PVA-borax gel and sol may suggest that the gel mode originates from a temporal network owing to chain entanglements. This behavior is very similar to that observed at the crossover from dilute to semidilute polymer solutions $[37,38]$. Thus cross-linking through borax is not necessary for the gel mode, but overlap of polymer chains or entanglements must be essential.

In order to confirm the prediction, we also performed NSE measurements on a PVA solution with the concentration of 4.8 wt \% without borax. The evaluated first cumulant or the initial decay rate $\Gamma_{\mathrm{i}}$ is plotted in Fig. 8 where $\Gamma_{\mathrm{i}}$ of the PVA-borax gel is also plotted for comparison. The value of the decay rate $\Gamma_{\mathrm{i}}$ of the PVA solution is identical to that of the PVA-borax gel. The $Q$ dependence of $\Gamma_{\mathrm{i}}$ is also identical: $\Gamma_{\mathrm{i}}$ is proportional to $Q^{2}$ and $Q^{3}$ below and above the critical $Q_{\text {c }}$, respectively, showing the crossover between the Zimm mode and the gel mode. These results suggest that the crosslinking points due to borax do not play an important role in the dynamics in the present $Q$ range although the macroscopic viscosity of the PVA-borax gel is dominated by the cross-linking owing to the borax. We also calculated the overlap concentration $C^{*}$ of the PVA chain [39], assuming that the persistence length is $8 \AA$ [40], and found that $C^{*}$ is $1.62 \mathrm{wt} \%$. This is lower than the PVA concentration of the sol (2.4 wt \%) in the NSE measurement, suggesting that polymer chains in the PVA-borax sol are overlapped each other, and hence the gel mode is observed in the PVA-borax sol with $C_{\mathrm{p}}=2.4 \mathrm{wt} \%$.

Finally we briefly consider the reason why the polymer chain motion in the gel, similar to the sol, is described by the Zimm model. As mentioned above, the macroscopic viscosity of the gel is more than 5 orders of magnitude larger than that of the sol, suggesting that an infinite network is formed through hydrogen bonds between PVA and borax and the macroscopic viscosity is dominated by the infinite network. If all borax molecules work as a cross-linker, one crosslinking point exists per 8.6 PVA monomers in average. In this situation, polymer chains must feel constraint owing to the cross-linking points. However, unexpectedly the NSE measurements show that polymer chains behave as if they were in solutions on the spatial scale of several $\mathrm{nm}$. This strongly suggests that not all borax molecules participate in the hydrogen bonds.

\section{CONCLUSION}

In this work, we have investigated dynamics of two types of PVA gels on a spatial scale of $\mathrm{nm}$ to tens $\mathrm{nm}$ using a neutron spin-echo technique. The observed normalized intermediate scattering function $I(Q, t) / I(Q, 0)$ of the PVA gel in a mixture of DMSO and water (60/40) consists of the fast decay, approximately below $3 \mathrm{~ns}$, and a nondecaying component. On the basis of the results the total SANS intensity was separated into the static (frozen) and dynamic fluctuations, indicating that the PVA gel is an immobile gel. The $Q$ dependence of the nondecaying component was analyzed in terms of the restricted motion in the network. On the other hand, the dynamics of the PVA gel in an aqueous borax solution (PVA-borax gel) is very different. $I(Q, t) / I(Q, 0)$ of the PVA-borax gel does not show the nondecaying component, similar to the PVA-borax sol, and is well described by the Zimm model, indicating that the PVA chains in the gel behave as if they were in solution. Surprisingly, the initial decay rate $\Gamma_{i}$ of the gel is identical to the sol on comparison at equal PVA concentrations while the macroscopic viscosity of the gel is more than 5 orders of magnitude larger than the sol. This was explained by not all the borax molecules participating in the hydrogen bonds, which form the crosslinking points. The NSE studies on the two PVA gels have demonstrated quite different dynamics and revealed the roles of cross-linking points in the gel dynamics. To better understand the role of cross-links and their influence in polymer gel we are currently investigating chemically cross-linked PVA gels. 
[1] Polymer Gels: Fundamentals and Biomedical Applications, edited by D. DeRossi, K. Kajiwara, Y. Osada, and A. Yamaguchi (Plenum, New York, 1991).

[2] Polyelectrolyte Gels: Properties, Preparation and Applications, edited by R. S. Harland and R. K. Prud'homme (American Chemical Society, Washington, DC, 1992).

[3] Gel Handbook, edited by Y. Osada and K. Kajiwara (Academic, Tokyo, 2001).

[4] J. Bastide and S. J. Candau, in Physical Properties of Polymeric Gels, edited by J. P. C. Addad (Wiley, Chichester, 1996), p. 143.

[5] E. Pines and W. Prins, Macromolecules 6, 888 (1973).

[6] K. Dusek and W. Prins, Fortschr. Hochpolym.-Forsch. 6, 1 (1969).

[7] M. Shibayama, Macromol. Chem. Phys. 199, 1 (1998).

[8] M. Shibayama and T. Norisuye, Bull. Chem. Soc. Jpn. 75, 641 (2002).

[9] T. Kanaya, M. Ohkura, K. Kaji, M. Furusaka, and M. Misawa, Macromolecules 27, 5609 (1994).

[10] T. Kanaya, H. Takeshita, Y. Nishikoji, M. Ohkura, K. Nishida, and K. Kaji, Supramol. Sci. 5, 215 (1998).

[11] T. Kanaya, M. Ohkura, H. Takeshita, K. Kaji, M. Furusaka, H. Yamaoka, and G. D. Wignall, Macromolecules 28, 3168 (1995).

[12] H. Takeshita, T. Kanaya, K. Nishida, and K. Kaji, Macromolecules 32, 7815 (1999).

[13] H. Takeshita, T. Kanaya, K. Nishida, K. Kaji, M. Hashimoto, and T. Takahashi, Phys. Rev. E 61, 2125 (2000).

[14] T. Tanaka, in Dynamic Light Scattering, edited by R. Pecora (Plenum, New York, 1985), p. 347.

[15] E. Geissler, in Dynamic Light Scattering, edited by W. Brown (Clarendon, Oxford, 1993), p. 471.

[16] T. Tanaka, L. O. Hocker, and G. B. Benedek, J. Chem. Phys 59, 5151 (1973).

[17] Neutron Spin Echo, Lecture Notes in Physics, Vol. 128, edited by F. Mezei (Springer-Verlag, Berlin, 1980).

[18] R. Oeser, B. Ewen, D. Richter, and B. Farago, Phys. Rev. Lett. 60, 1041 (1988).

[19] A. M. Hecht, F. Horkay, P. Schleger, and E. Geissler, Macromolecules 35, 8552 (2002).

[20] T. Kanaya, N. Takahashi, K. Nishida, K. Kaji, H. Seto, M.
Nagao, Y. Kawabata, and T. Takeda, J. Neutron Res. 10, 149 (2002).

[21] S. Koizumi, M. Monkenbusch, D. Richter, D. Schwahn, B. Farago, and M. Annaka, J. Neutron Res. 10, 155 (2002).

[22] H. Deuel and H. Neukom, Makromol. Chem. 3, 13 (1949).

[23] M. Shibayama, M. Sato, H. Kurokawa, and S. Nomura, Polymer 29, 336 (1988).

[24] A. Koike, N. Nemoto, T. Inoue, and K. Osaki, Macromolecules 28, 2339 (1995).

[25] N. Nemoto, A. Koike, and K. Osaki, Macromolecules 29, 1445 (1996).

[26] A. Takada and N. Nemoto, Prog. Colloid Polym. Sci. 106, 183 (1997).

[27] A. Takada, M. Nishimura, A. Koike, and N. Nemoto, Macromolecules 31, 436 (1998).

[28] T. Inoue and K. Osaki, Rheol. Acta 32, 550 (1993).

[29] K. H. Ahn and K. Osaki, J. Non-Newtonian Fluid Mech. 55, 215 (1994).

[30] K. Osaki, T. Inoue, and K. H. Ahn, J. Non-Newtonian Fluid Mech. 54, 109 (1994).

[31] Y. Ito, M. Imai, and S. Takahashi, Physica B 213/214, 889 (1995).

[32] T. Takeda, H. Seto, S. Komura, S. K. Ghosh, M. Nagao, J. Matsuba, H. Kobayashi, T. Ebisawa, S. Tasaki, C. M. E. Zeyen, Y. Ito, S. Takahashi, and H. Yoshizawa, J. Phys. Soc. Jpn. 65, 189 (1996).

[33] T. Springer, Quasielastic Neutron Scattering for the Investigation of Diffusive Motions in Solids and Liquids (SpringerVerlag, Berlin, 1972).

[34] B. H. Zimm, J. Chem. Phys. 24, 269 (1965).

[35] B. Ewen and D. Richter, Adv. Polym. Sci. 134, 1 (1997).

[36] P. G. de Gennes and E. Dubois-Violette, Physics (Long Island City, N.Y.) 3, 181 (1967).

[37] D. Richter, J. B. Hayter, F. Mezei, and B. Ewen, Phys. Rev. Lett. 41, 1484 (1978).

[38] B. Ewen, D. Richter, J. B. Hayter, and B. Lehnen, J. Polym. Sci., Polym. Lett. Ed. 20, 233 (1982).

[39] M. Ohkura, T. Kanaya, and K. Kaji, Polymer 33, 3689 (1992).

[40] M. Kurata and Y. Tsunashima, in Polymer Handbook, edited by J. Brandrup and E. H. Immergut (Wiley Interscience, New York, 1989), p. VII/36. 